\title{
Stress Corrosion Cracking Behavior of Fine-Grained AZ61 Magnesium Alloys Processed by Equal-Channel Angular Pressing
}

\author{
Qiuyuan Xie ${ }^{1}$, Aibin Ma ${ }^{1,2, *}$, Jinghua Jiang ${ }^{1}$, Zhaojun Cheng ${ }^{1}$, Dan Song ${ }^{1}$, Yuchun Yuan ${ }^{1}$ and \\ Huan Liu ${ }^{1}$ \\ 1 College of Mechanics and Materials, Hohai University, Nanjing 210098, China; xqywaly@163.com (Q.X.); \\ jinghua-jiang@hhu.edu.cn (J.J.); chengzj125@163.com (Z.C.); songdancharls@hhu.edu.cn (D.S.); \\ ycyuan@hhu.edu.cn (Y.Y.); liuhuanseu@hhu.edu.cn (H.L.) \\ 2 Suqian Institute, Hohai University, Suqian 223800, China \\ * Correspondence: aibin-ma@hhu.edu.cn; Tel.: +86-258-378-7239; Fax: +86-258-378-6046
}

Received: 21 July 2017; Accepted: 25 August 2017; Published: 4 September 2017

\begin{abstract}
The effect of equal-channel angular pressing (ECAP) on stress corrosion cracking (SCC) behavior of a cast AZ61 Mg alloy was investigated in distilled water (DW) using the slow strain rate tensile test (SSRT) at a strain rate of $1 \times 10^{-6} \mathrm{~s}^{-1}$. The fine-grained alloy after ECAP showed a greater SCC susceptibility but a higher ultimate tensile strength, compared with the as-cast counterpart. The results were attributed to refined grains, high-density dislocations and increased proportion of high-angle grain boundaries induced by severe plastic deformation, as well as isolated fine $\beta$-phase particles transiting from net-like $\beta$-phase.
\end{abstract}

Keywords: magnesium alloy; stress corrosion cracking; ECAP; fine-grained

\section{Introduction}

Magnesium alloys are very attractive light materials, due to a high strength to weight ratio, being pollution-free, non-toxic, and exhibiting easy recovery [1,2], etc. The applications of magnesium alloys in electronics, automobiles, aerospace and the biomedical field are rapidly increasing, but their high chemical reactivity and poor corrosion resistance still severely limit their wider application $[3,4]$. In particular, magnesium alloys are susceptible to stress corrosion cracking because of the synergy between mechanical stress and the corrosion medium during their service as engineering structural materials, with heavy economic losses due to unexpected premature failure. Therefore, the pressing matter of the moment is to understand the SCC behavior of magnesium alloys so as to improve their SCC resistance, thus making greater application of structural components of magnesium alloys possible.

Constant efforts have been made to figure out the influencing factors of SCC behavior of magnesium alloys, including alloy composition, the manufacturing process, microstructure and heat treatment [1], etc. Furthermore, it has been demonstrated [5] that hydrogen is crucial to the mechanism of the SCC of magnesium alloys. Based on these, many investigations have been carried out to obtain less susceptibility to SCC of Mg alloys. Remarkable improvement has been achieved in some reports, but research on this matter is far from conclusive. Tomashov and Modestova [6] indicated that the susceptibility of Mg-Al-Mn alloys increased with small additions of Ce, compared to the improved SCC resistance of EV31A by the additions of $\mathrm{Nd}$ or $\mathrm{Zr}$ [7]. Srinivasan et al. [4] reported that the wrought AZ61 Mg alloy processed by plasma electrolytic oxidation improved general corrosion resistance to a significant extent, attributed to the protection of plasma electrolytic oxidation coating on the specimen surface, yet the enhancement in the resistance to SCC was much less distinct. 
Severe plastic deformation (SPD), as a well-known method of manufacturing fine-grained metals and alloys, is a promising way to improve the SCC resistance of Mg alloys, accompanied by the enhancement of both strength and ductility. Available study [8] showed that the general corrosion resistance of Mg alloys was definitely improved after grain refinement via SPD. However, a conclusion about the effect of grain refinement on SCC has not yet been reached in the limited literature. Argade et al. [9] reported that ultrafine grained AZ31 processed by friction stir processing, one of the SPD methods, exhibited higher susceptibility to SCC because of the enhanced hydrogen diffusivity. Lopez et al. [10] claimed that an austenitic stainless steel containing $0.97 \mathrm{wt} \%$ nitrogen achieved higher resistance to SCC through grain refinement compared to the as-received steel in $30 \% \mathrm{NaCl}$ solution at $90^{\circ} \mathrm{C}$.

In the present work, AZ61 Mg alloy has been chosen as the experimental sample, fabricated by equal-channel angular pressing to get a fine-grained microstructure. All Al-containing magnesium alloys have been confirmed to be susceptible to SCC in distilled water [1]. Generally, the SCC susceptibility of $\mathrm{Mg}$ alloys tends to increase with the growing percentage of $\mathrm{Al}$ content [11], and a previous study even showed the SCC susceptibility achieved maximize with about $6 \% \mathrm{Al}$ content [12]. Herein, the fine-grained AZ61 samples were fabricated by multi-pass ECAP and then the stress corrosion resistance was investigated by slow strain rate tensile tests (SSRT) in air and distilled water, respectively. SSRT is considered an advantageous testing method, in which the SCC susceptibility of samples could be easily evaluated [2]. The present work gives an insight into the relationship among temperature, grain size and structural homogeneity so as to discuss the influence of processed temperature on the SCC behavior of samples. The objective of current research is to investigate the influence of grain refinement on the SCC behavior of AZ61 Mg alloy.

\section{Experimental Procedures}

\subsection{Material}

The material used in the present study is AZ61 magnesium alloy. The billets for ECAP $(20 \mathrm{~mm} \times 20 \mathrm{~mm} \times 45 \mathrm{~mm})$ were prepared by electrospark wire-electrode cutting. Then different bulk ultrafine-grained samples were obtained using a self-made ECAP die $\left(\Phi=90^{\circ}, \Psi=0^{\circ}\right)$, meanwhile optimizing parameters of the ECAP process to produce the high-quality samples without cracks. Different passes and extrusion temperature of ECAP were applied, i.e., 8 passes at $673 \mathrm{~K}, 16$ passes at $673 \mathrm{~K}$, and 16 passes at $623 \mathrm{~K}$, respectively. Hereinafter, the as-cast and ECAP processed (ECAPed) AZ61 samples of 8-pass processed at $673 \mathrm{~K}, 16$-pass processed at $673 \mathrm{~K}$ and 16-pass processed at $623 \mathrm{~K}$ are designated as $1 \#, 2 \#, 3 \#$, and $4 \#$, respectively.

\subsection{Slow Strain Rate Tensile Testing}

All samples were tensioned on a slow strain rate tensile testing machine (RGM-4050, Reger, Shenzhen, China) to investigate the SCC behaviors of AZ61 Mg alloy. In this case, air and distilled water were used as test mediums, and all tests were done at room temperature. Load was imposed on samples through the shifting cross-head of the testing device at quite slow but constant speed, followed by the accelerated initiation of SCC due to the strengthened strain state. Figure 1 shows details of tensile samples, of which the axial direction was parallel to the extrusion direction, and the thickness of samples was $2 \mathrm{~mm}$. To rule out the effects of roughness on tests, all samples were abraded along the tensile direction with silicon carbide sandpaper up to 2000 grit. SSRT was carried out at a strain rate of $1 \times 10^{-6} \mathrm{~s}^{-1}$ and a pre-load of $50 \mathrm{~N}$ was applied before trails. Cleaning of fractured samples was performed using absolute alcohol in an ultrasonic cleaner immediately after the failure and then samples were dried in a vacuum oven for the observation of fracture morphology. 


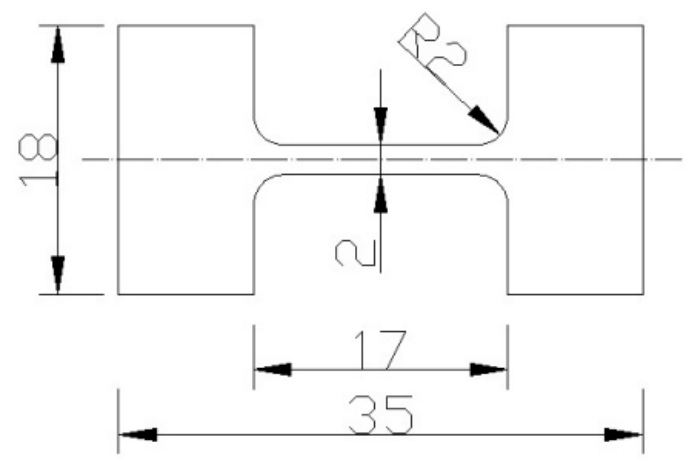

Figure 1. Diagram of AZ61 Mg alloy samples for slow strain rate tensile test (SSRT) (unit: mm).

To evaluate the SCC resistance of the Mg alloy, the SCC susceptibility index (ISCC) for each sample was established, based on the absorbed energy $(A)$ before its disruption, in air or in distilled water, and is defined as [1]:

$$
I_{\mathrm{SCC}}=\frac{A_{\mathrm{air}}-A_{\mathrm{dw}}}{A_{\mathrm{air}}} \times 100 \%
$$

where $A_{\mathrm{air}}$ and $A_{\mathrm{dw}}$ are the value of absorbed energy, calculated according to the areas under stress-strain curves [1] in air and in distilled water, respectively. The greater value of $I_{\mathrm{SCC}}$ corresponds to the stronger SCC tendency.

\subsection{Microstructure Observation}

The microstructure of the as-cast and the ECAPed AZ61 samples was investigated along with the extrusion direction by a OLYMPUS-BX51M optical microscope (OM, Olympus, Tokyo, Japan). Previously, all samples were abraded with $\mathrm{SiC}$ sandpaper up to 2000 grit, prepared by mechanical polishing, and then etched in the mixture of $10 \mathrm{~mL}$ acetic acid, $3 \mathrm{~g}$ picric acid, $25 \mathrm{~mL}$ ethanol, and $10 \mathrm{~mL}$ distilled water. Energy dispersive spectroscope (EDS, Bruker, Beijing, China) was carried out on four types of samples to reveal the species and distribution of elements, and then it provided a preliminary analysis of the secondary phase or precipitate distribution accordingly. For further specifications, transmission electron microscope (TEM, FEI, Hillsboro, OR, USA) observation was conducted to present information on crystal structure, including grain size, dislocation, twinning, etc. Samples for TEM were prepared by means of ion beam-thinning. Fractography of fractured samples after SSRT was performed using scanning electron microscope (SEM, Hitachi, Tokyo, Japan) for the study of evolution of SCC behaviors of the as-cast and the ECAPed AZ61 samples.

\section{Results}

\subsection{Microstructure}

Figure 2 shows optical micrographs of the as-cast and various ECAPed AZ61 samples of 8 passes at $673 \mathrm{~K}, 16$ passes at $673 \mathrm{~K}$, and 16 passes at $623 \mathrm{~K}$, respectively. The microstructure of as-cast AZ61 alloy possessed a coarse $\alpha$-phase matrix and secondary phase or $\beta-\mathrm{Mg}_{17} \mathrm{Al}_{12}$ intermetallic, primarily along the grain boundaries. The average grain size of the as-cast alloy was $\sim 100 \mu \mathrm{m}$, determined by linear intercept method. Significantly structural deformations were presented in the ECAPed AZ61 samples. Grains were refined to the average size of $\sim 10 \mu \mathrm{m}$ after $8 \mathrm{ECAP}$ passes at $673 \mathrm{~K}$, whereas the microstructure was generally inhomogeneous and there were still coarse grains. After 16 passes at $673 \mathrm{~K}$, the microstructural uniformity deteriorated compared to the 8-pass AZ61 alloy processed at the same temperature, with the refined grains distributed along the extruded direction and the remaining existing large grains, and the value of average grain size was $\sim 15 \mu \mathrm{m}$. Figure $2 \mathrm{~d}$ indicates that grains were refined appreciably, exhibiting relative homogeneity, and deformation bands composed of refined grains distributed along the extruded direction were formed. Here the average size was $\sim 5 \mu \mathrm{m}$. 
Compared to the ECAPed Al alloys, the grains of ECAPed AZ61 Mg alloys were fairly coarse [13], primarily due to the difference in processing temperature of ECAP. The processing temperature of $\mathrm{Al}$ (ordinarily less than $423 \mathrm{~K}$ ) is much lower than that of the AZ61 alloy $[13,14]$.
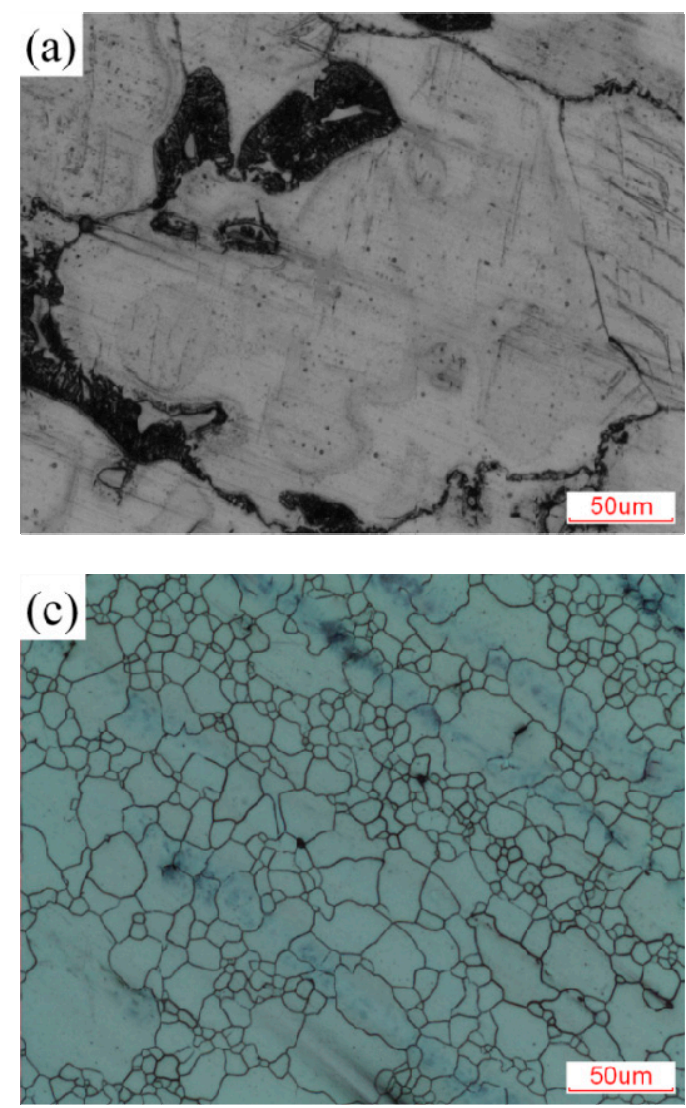
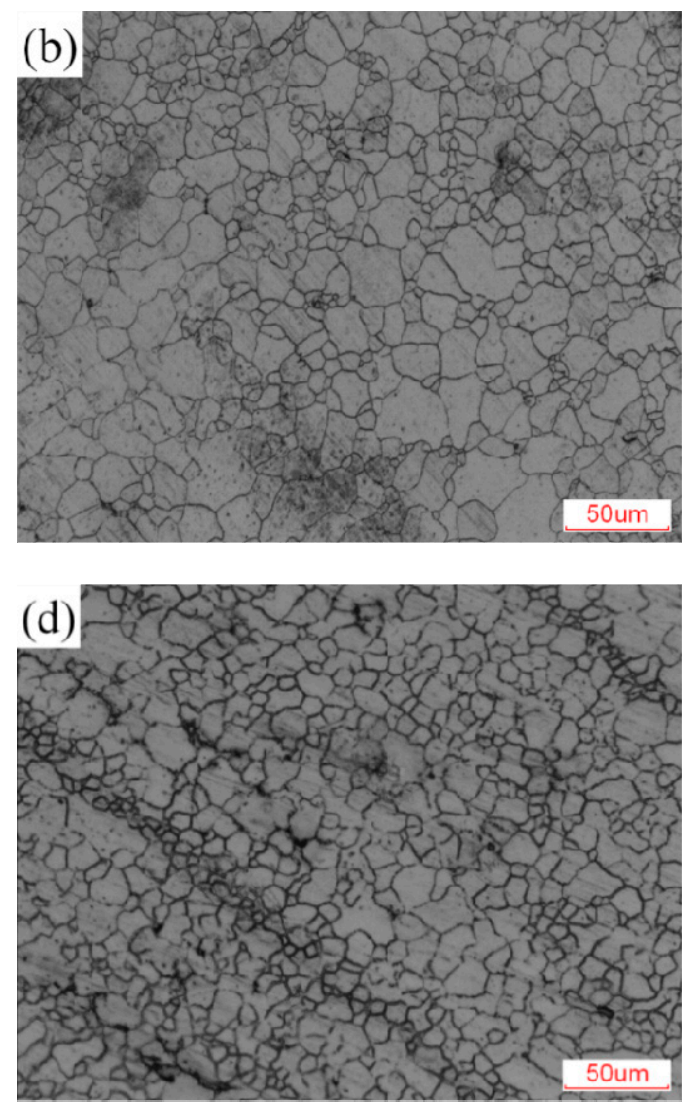

Figure 2. Optical micrographs of as-cast and ECAP processed (ECAPed) AZ61 Mg alloy (a) 1\#, (b) 2\#, (c) $3 \#$ and (d) $4 \#$.

Figure 3 shows the TEM micrographs of the as-cast and various ECAPed AZ61 samples for 8 passes at $673 \mathrm{~K}, 16$ passes at $673 \mathrm{~K}$, and 16 passes at $623 \mathrm{~K}$. The relatively heterogeneous microstructure of as-cast AZ61 Mg alloy was the mixture of large grains with deformation bands and twins accompanied with low dislocation density and high fraction of low-angle grain boundaries. After ECAP, the microstructure homogeneity was improved significantly. According to the ring-shaped selected area electron diffraction (SAED) pattern of ECAPed samples, grains were remarkably refined and the proportion of high-angle grain boundaries increased accordingly. The growing misorientation angles were consistent with the consequence of dominant process (dynamic recrystallization process) in other ECAPed metals $[15,16]$. A fairly high density of tangled dislocations was formed, notably in $3 \#$ samples. Figure 4 shows the element maps of $\mathrm{Mg}$ and $\mathrm{Al}$ overlapped on EDS images, providing a rough idea for the distribution of the $\beta-\mathrm{Mg}_{17} \mathrm{Al}_{12}$. The green and red patches represented $\mathrm{Mg}$ and $\mathrm{Al}$, respectively. It can be speculated that the majority of the red particles were $\mathrm{Mg}_{17} \mathrm{Al}_{12}$. By contrast, the coarse $\beta$-phase in as-cast AZ61 revealed appreciable refinement into uniformly dispersed fine particles with trace large particles retained after ECAP, as shown in Figure 4 indicated by arrows. Compared to the 16-pass samples processed at $673 \mathrm{~K}$ or $623 \mathrm{~K}$, the refinement was less significant for the 8-pass sample, attributed to the lower ECAP passes. 

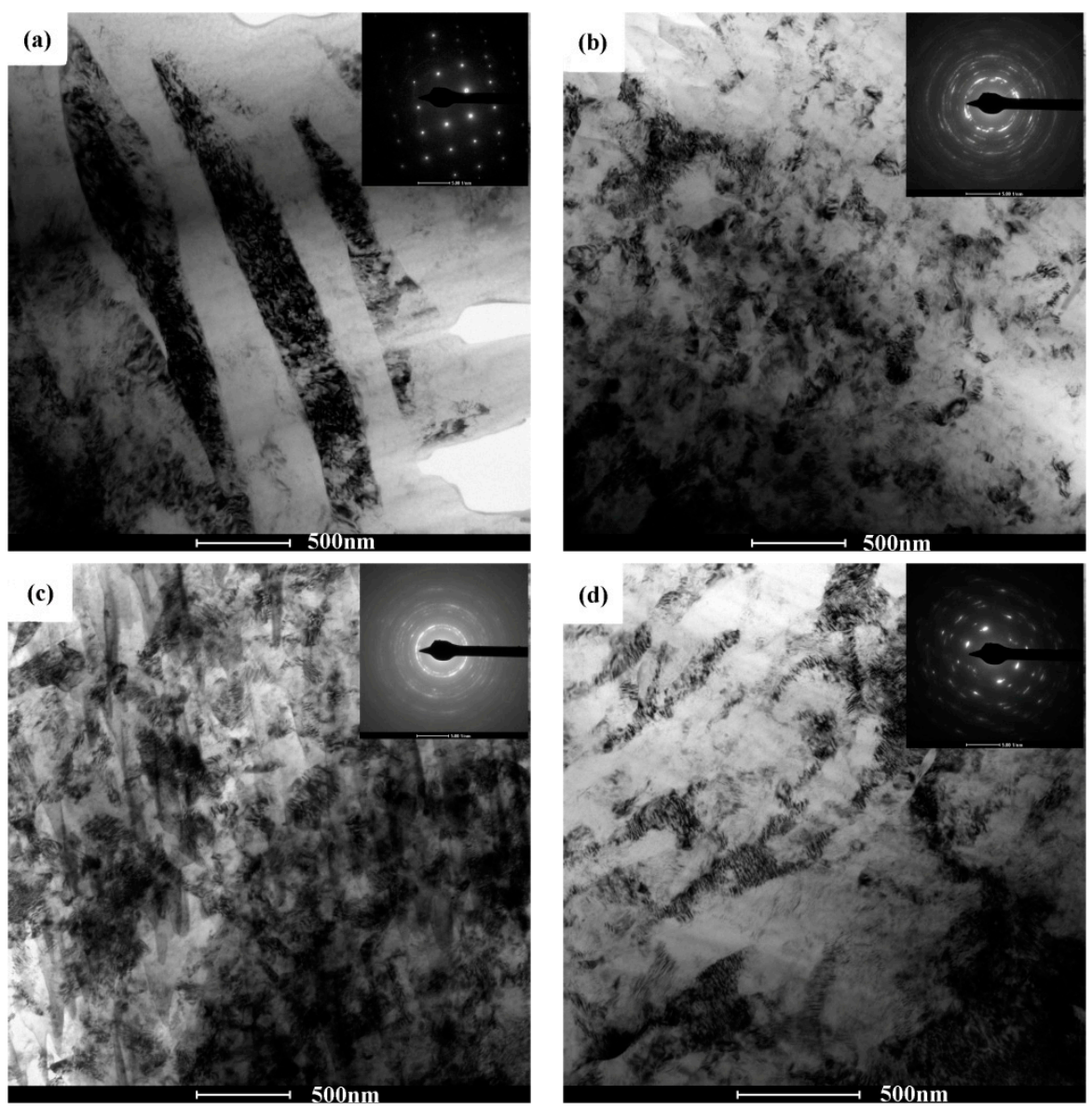

Figure 3. TEM images and corresponding selected area electron diffraction (SAED) patterns of various AZ61 magnesium alloys: (a) 1\#, (b) 2\#, (c) 3\# and (d) 4\#.
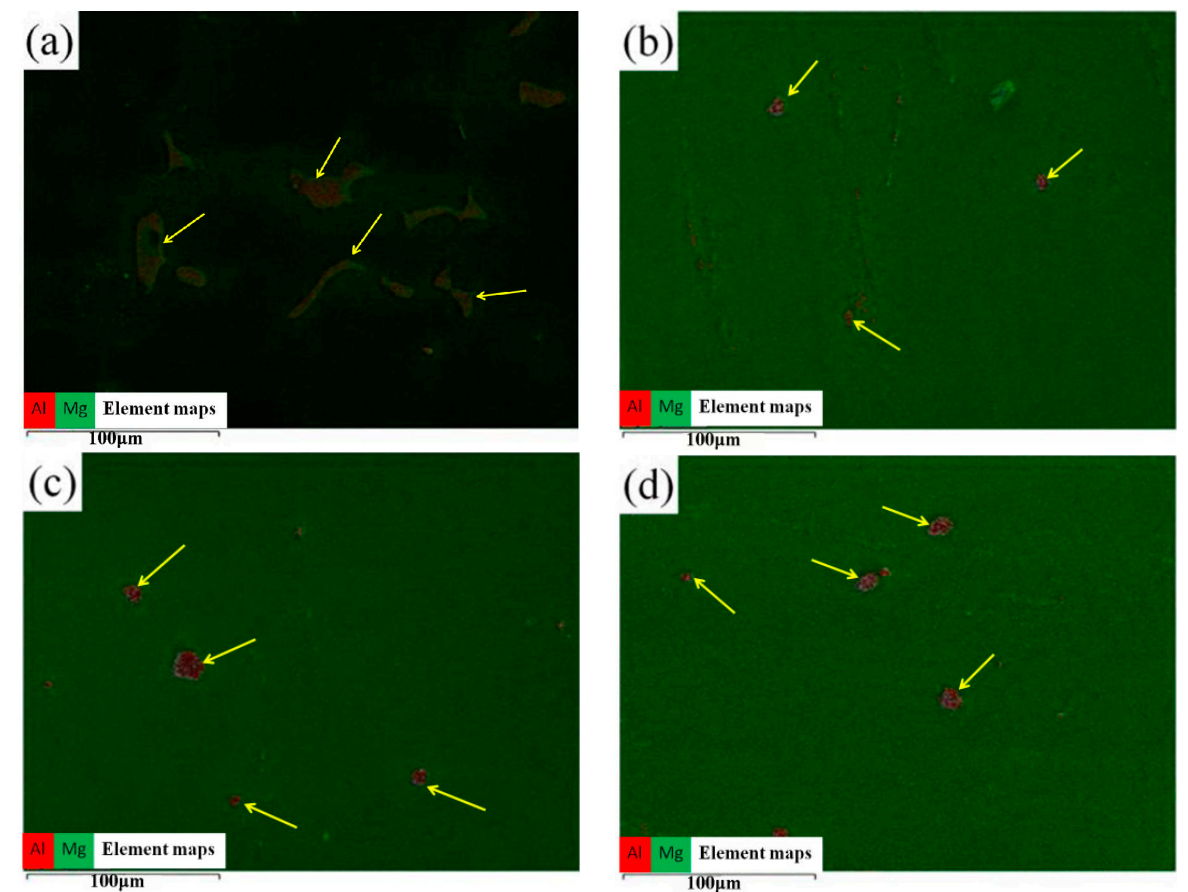

Figure 4. Element maps of $\mathrm{Mg}$ and $\mathrm{Al}$ overlapped on energy dispersive spectroscope (EDS) images: (a) 1\#, (b) 2\#, (c) 3\# and (d) 4\# (arrows indicate $\beta$-phase particles). 


\subsection{Slow Strain Rate Tensile Testing}

The typical SSRT stress-strain curves for the studied alloys are presented in Figure 5. Table 1 shows the value of yield strength, ultimate tensile strength, elongation, absorbed energy to failure and SCC indices for the four different samples. Apparently, the yield strength and ultimate tensile strength were enhanced after ECAP processing compared to the as-cast one, largely originating from the strengthening effect of the refined grains [17]. In addition, 4\# samples showed superior properties, a combination of strength and elongation in both air and distilled water, compared to the other two samples processed by ECAP. The relatively advantageous performances of $4 \#$ samples are primarily attributed to grain refinement and the comparatively homogeneous structure. A remarkable similarity among all the samples is the significant loss of ultimate tensile strength and elongation when tested in distilled water compared to air.

The susceptibility indices of SCC are calculated based on Equation (1). It is quite noticeable that the as-cast AZ61 Mg alloy exhibited high SCC susceptibility with a SCC index of $42.50 \%$ in distilled water. After ECAP processing, all the ECAPed samples showed higher SCC susceptibility indices, indicating a greater tendency of SCC. Additionally, 2\# samples showed a relatively low value of SCC index and there is a large difference with the other two ECAPed alloys. Moreover, 8-pass samples had the distinctly lower SCC susceptibility indices compared to the 16-pass samples fabricated at the same temperature. Hence, it suggests that processing passes and temperature play predominant roles in SCC behaviors of AZ61 Mg alloy. Besides, there is a phenomenon that stress jumped at constant strain, known as the "pop-in" phenomenon, probably due to the local embrittlement at crack tips because of the presence of impurity defects or secondary phases in material [18].
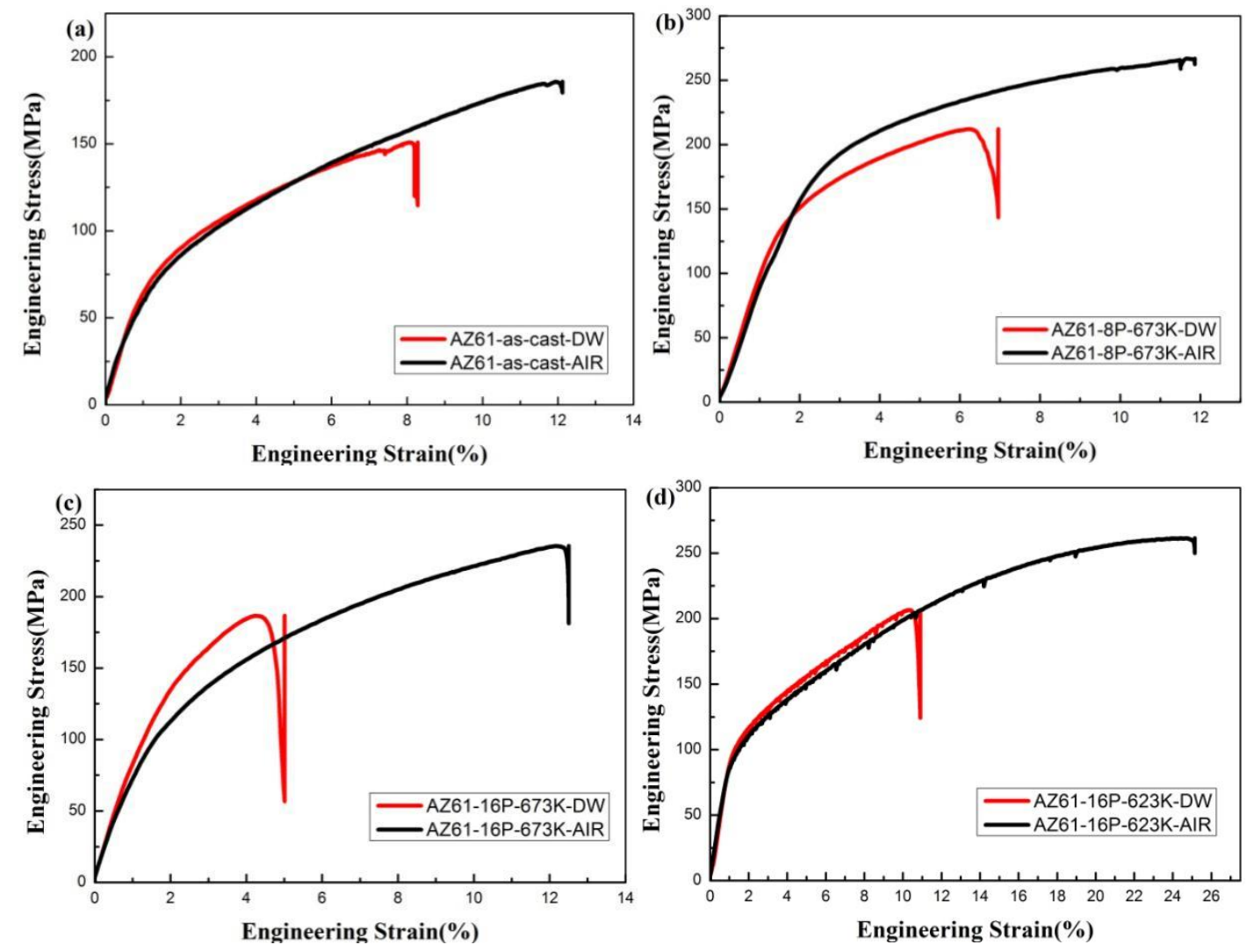

Figure 5. Typical SSRT curves of various of AZ61 Mg alloys tested in air and distilled water (DW) (a) 1\#, (b) 2\#, (c) 3\# and (d) 4\#. 
Table 1. Mechanical properties and stress corrosion cracking (SCC) indices for the studied alloys.

\begin{tabular}{|c|c|c|c|c|c|c|}
\hline Alloy & Test Medium & $\begin{array}{c}\text { Yield } \\
\text { Strength (MPa) }\end{array}$ & $\begin{array}{l}\text { Ultimate Tensile } \\
\text { Strength (MPa) }\end{array}$ & Elongation (\%) & $\begin{array}{l}\text { Absorbed Energy } \\
\text { to Failure }\left(\mathrm{KJ} / \mathrm{m}^{3}\right)\end{array}$ & $\begin{array}{l}\text { SCC Susceptibility } \\
\text { Index, I ISC (\%) }\end{array}$ \\
\hline \multirow[b]{2}{*}{$1 \#$} & AIR & 64 & 186 & 12.12 & $15,805.21$ & \multirow[b]{2}{*}{42.50} \\
\hline & DW & 71 & 151 & 8.28 & 9087.45 & \\
\hline \multirow[b]{2}{*}{$2 \#$} & AIR & 153 & 267 & 11.86 & $24,760.22$ & \multirow{2}{*}{54.83} \\
\hline & DW & 138 & 212 & 6.96 & $11,184.32$ & \\
\hline \multirow[b]{2}{*}{$3 \#$} & AIR & 106 & 235 & 12.50 & $21,401.24$ & \multirow{2}{*}{69.66} \\
\hline & DW & 118 & 186 & 5.01 & 6492.35 & \\
\hline \multirow[b]{2}{*}{$4 \#$} & AIR & 96 & 261 & 25.14 & $50,594.78$ & \multirow{2}{*}{67.45} \\
\hline & DW & 105 & 206 & 10.77 & $16,467.80$ & \\
\hline
\end{tabular}

\subsection{Fractography}

For further investigation of the susceptibility indicated by SSRT results, the SEM fractographies of all studied samples in air and in distilled water are reported in Figure 6. The fracture surfaces of the as-cast sample that failed in air and in distilled water are shown in Figure $6 a, b$, respectively. Figure $6 a$ reveals mixed mode with transgranular cleavage and dimpled features, as well as intergranular fracture, mainly initiated by preferential anodic dissolution of the matrix adjacent to $\beta-\mathrm{Mg}_{17} \mathrm{Al}_{12}$ [1], whereas the same sample tested in distilled water showed a predominantly transgranular feature with parallel facets, attributed to hydrogen-assisted embrittlement for the AZ series alloys $[5,19,20]$. The primary conclusion is that the as-cast AZ61 Mg alloy behaved fairly susceptible to SCC in distilled water. The fracture morphology of 2\# samples was distinctly different. For fracture surface of 2\# sample tested in distilled water, shown in Figure 6c, intergranular features coexisted with slightly transgranular cleavage, as well as dimpled structures. The fracture surface of $3 \#$ sample is presented in Figure $6 \mathrm{~d}$, when tested in distilled water. Transgranular cleavage-like facets are observed in Figure $6 \mathrm{~d}$, moreover, there were transgranular cracks accompanied by a few secondary cracks associated with plastic accommodation of the hydride [21], suggesting the distinctive susceptibility to SCC of 3\# sample in distilled water. Remarkably, fractures in Figure 6e and $\mathrm{f}$ showed quite different features from the above. When $4 \#$ samples were tested in air, the fracture showed fine and dense dimples with few cleavage facets. For the sample tested in distilled water, dimpled features severely dropped and definite cleavage planes developed, suggesting that $4 \#$ samples underwent enormous losses in ductility and therefore showed intensive severity of SCC susceptibility in distilled water compared to the as-cast one.
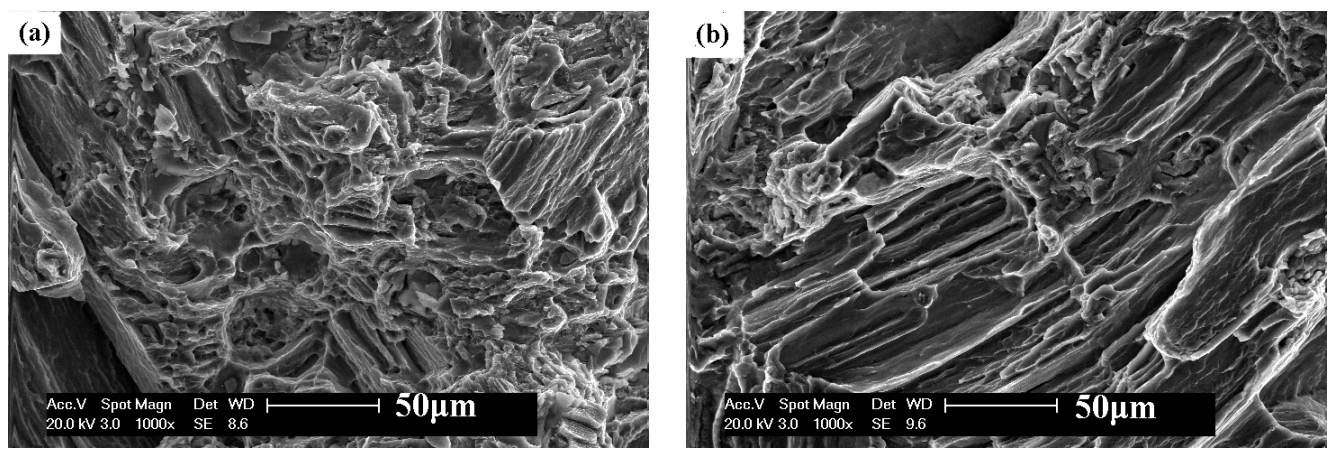

Figure 6. Cont. 

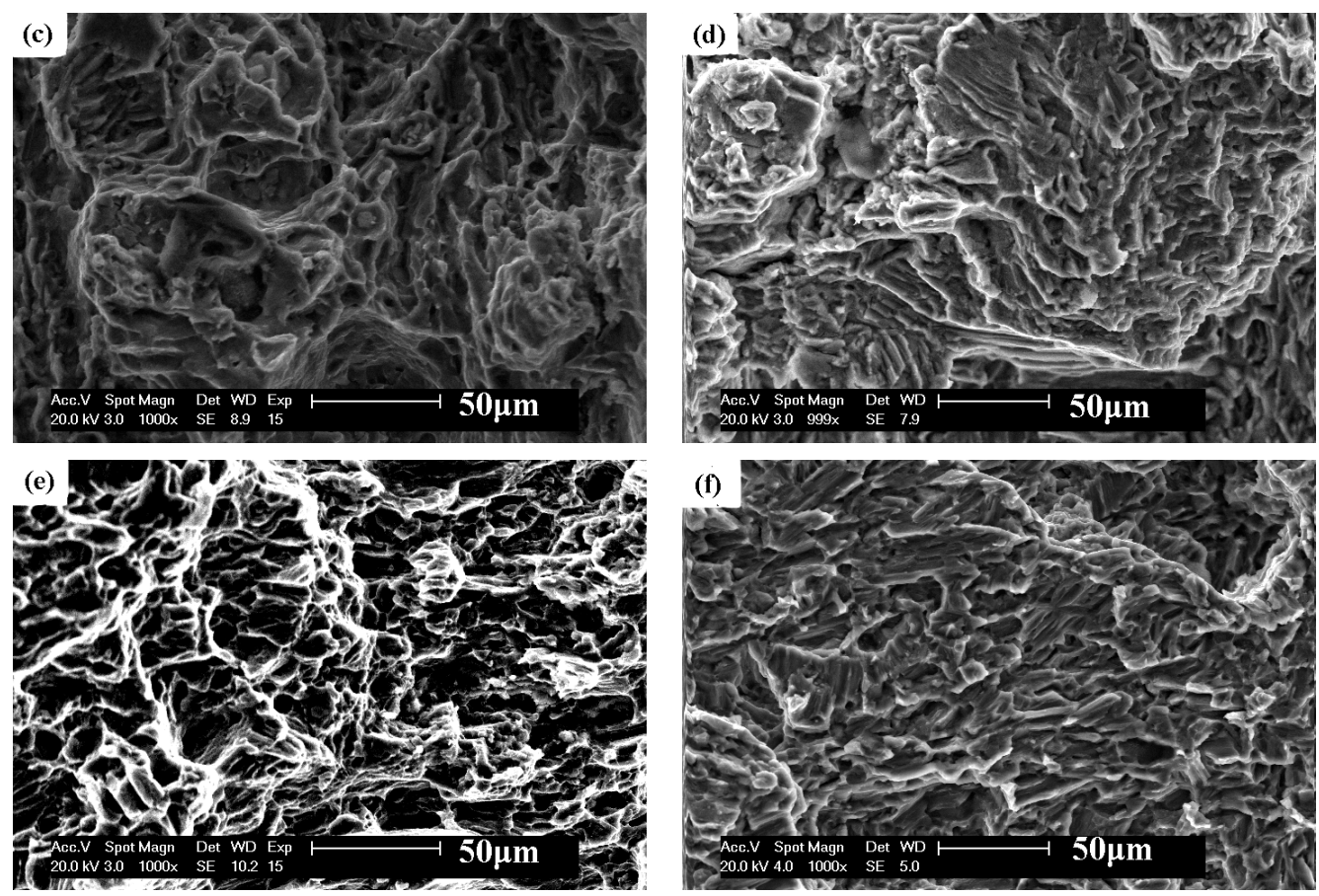

Figure 6. SEM fractography of the SSRT test samples (a) $1 \#$ in air, (b) $1 \#$ in DW, (c) 2\# in DW, (d) $3 \#$ in DW, (e) 4 \# in air and (f) 4 \# in DW.

\section{Discussion}

A conclusion about the effects of grain refinement on SCC behaviors has not been reached yet in the limited literature. In the present study, all of the results, mainly SSRT measurements, revealed higher ultimate tensile strength but greater SCC susceptibility of the refined samples via ECAP in distilled water. The behaviors could be attributed to the evolution of microstructure, defects and secondary phase in the process of ECAP.

The mechanism of grain refinement for ECAP should be the synergistic effect of mechanical shear, dynamic recovery and dynamic recrystallization. During the continuous ECAP process, large quantities of dislocations were generated, tangled and then converted into dislocation cells and walls, breaking up coarse grains into subgrain boundaries to develop the refined grains. The growth of recrystallized grains would be promoted at a relatively high temperature, especially over the recrystallization temperature, as well as in the excessive runtime of ECAP processing. Hence, it seems reasonable that 16-passed sample fabricated at $623 \mathrm{~K}$ possessed a fairly fine and homogeneous microstructure. This indicates that temperature may play a more significant role in grain refinement than processing pass. For the relatively low stacking default energy of $\mathrm{Mg}$ matrix [22], there were still considerable dislocations tangled in the matrix as seen from Figure $3 b-d$, despite the dynamic recovery and recrystallization for the duration of ECAP; more processing passes may lead to higher density of accumulated dislocations. Increased fraction of high-angle boundaries were observed following ECAP processing. After continuously multi-pass ECAP, coarse net-like eutectic of $\beta-\mathrm{Mg}_{17} \mathrm{Al}_{12}$ was gradually elongated and broken to form isolated and finer particles [23]. Moreover, ultimate tensile strength was enhanced for the fine grain microstructure in accordance with the Hall-Petch formula. It follows that lower processing temperature favored grain refinement so as to obtain higher ultimate tensile strength, whereas ultimate tensile strength decreased following ECAP processing at a relatively high temperature of $673 \mathrm{~K}$ in a comparison between 8-pass sample and 16-pass one.

Since the AZ61 developed a mixed fracture mode of intergranular SCC and transgranular SCC, cracks were facilitated by a combined effect of anodic dissolution and hydrogen-assisted embrittlement. The mechanism for crack propagation involves the preferential attack of the anodic 
matrix and repetitive formation and fracture of a brittle zone simultaneously [21]. Some research has identified that grain refinement can achieve the improved general corrosion resistance and ease local corrosion [24]. It is believed that grain boundaries can act as a barrier to the development of corrosion [25]. Grain refinement brought more grain boundaries, contributing to the higher corrosion resistance compared to the coarse one. Research showed that the initial film layer formed on $\mathrm{Mg}$ - $\mathrm{Al}$ alloys substrate would be $\mathrm{Al}_{2} \mathrm{O}_{3}$, accompanied by $\mathrm{MgO}$ outside covered with $\mathrm{Mg}(\mathrm{OH})_{2}$ in aqueous solution [26,27]. Higher fraction of oxide film was expected to form on the fine-grained sample. The propensity of the corrosion medium and hydrogen to enter into the matrix would drop considerably for the relatively more protective oxide film. Herein, general corrosion would be advantageous to the improvement of SCC resistance accordingly. Additionally, a fine-grained microstructure means the possibility of stress reliving to reduce stress concentration on surface film, otherwise leading to crack initiation on the surface and therefore accelerating the corrosion rate and diffusion of hydrogen ahead of the crack tips. Actually, the oxide film was not passive enough to provide protection for the substrate because of its porosity and instability. Nevertheless, grain boundaries can be a barrier to the crack propagation as well, in theory. Hence, SCC resistance might be improved for increased energy required for crack propagation when there are more grain boundaries per volume for the refined microstructure and differences in orientations between adjacent grains.

Crystal defects stored high energy, such as dislocations and high-angle grain boundaries, were introduced as well with the refined microstructure. Activation of corrosion would be promoted due to the high energy stored in the matrix. Hydrogen was inclined to be trapped by those defects and therefore gathered to form high pressure inside the matrix inducing crack initiation. Besides, grain boundaries, as a kind of crystal defect, stored high energy as well and exhibited unfavourable impact on corrosion resistance to some extent. Diffusion of hydrogen prone to grain boundaries and diffusivity will be accelerated with the increased proportion of grain boundaries per volume of refined microstructure [9].

An enormous amount of $\beta$-phase precipitation distributed along grain boundaries makes a significant difference in SCC behaviors of AZ61 Mg alloy. Reports claimed that $\beta$-phase played a role as cathode in galvanic corrosion coupling with $\alpha$-phase matrix [1,28]. In the meantime, the net-like $\beta$-phase could work as a barrier to the corrosion propagation of $\alpha$-phase matrix [23]. As shown in Figure 4 , net-like $\beta$-phase existed in the as-cast samples were refined into fine particles distributing uniformly in the matrix after ECAP processing. As a result, large quantities of micro-galvanics distributing homogeneously in the matrix formed, leading to uniform corrosion for the ECAPed AZ61 samples. $\alpha-\mathrm{Mg}$ matrix would be weakened by widespread destruction caused by micro-galvanics corrosion despite the "small cathode, large anode", and therefore accelerated anodic dissolution of matrix. Additionally, $\beta$-phase would assist rather than hinder corrosion propagation of $\alpha$-Mg matrix for the crumbled barrier action of net-like $\beta$-phase. Hence, the accelerated rupture of ECAPed samples for the refined $\beta$-phase precipitations can be seen.

Taken together, the increased SCC tendency of ECAPed AZ61 Mg alloys could be related to the combined effect of grain refinement, introduced defects and refined $\beta$-phase following ECAP processing. Although finer grains of the Mg matrix after multi-pass ECAP retarded cracks propagation to some extent, enormous crystalline defects introduced by severe plastic deformation accelerated corrosion destruction and diffusion of hydrogen along with isolated fine $\beta$-phase particles.

\section{Conclusions}

SCC behaviors of as-cast and ECAPed AZ61 Mg alloys were investigated by SSRT tests and the following conclusions could be made:

1. Fine-grained AZ61 Mg alloys were fabricated via multi-pass ECAP processing both at $623 \mathrm{~K}$ and $673 \mathrm{~K}$. The decrease in processing temperature is favourable to the refinement of the 
microstructure, resulting in the enhancement of ultimate tensile strength and even elongation in distilled water.

2. The as-cast AZ61 Mg alloys with about $6 \% \mathrm{Al}$ content have a great tendency to SCC in distilled water and the ECAPed processing increased the susceptibility to SCC at room temperature.

3. The greater SCC tendency of ECAPed AZ61 Mg alloys was related to the combined effect of grain refinement, introduced defects and refined $\beta$-phase following ECAP processing. Fine grains of the $\mathrm{Mg}$ matrix could retard cracks propagation to some extent, whereas enormous crystalline defects introduced by severe plastic deformation accelerated corrosion destruction and diffusion of hydrogen along with crumbled barrier action for a transition from net-like $\beta$-phase to isolated fine $\beta$-phase particles.

Acknowledgments: The study was supported by the National Natural Science Foundation of China (Grant No. 51774109), the Key Research and Development Project of Jiangsu Province of China (Grant No. BE2017148), the Fundamental Research Funds for the Central Universities (Grant No. HHU2016B10314), Six Major Talent Peaks Project of Jiangsu Province of China (Grant No. 2014-XCL-023), the Public Service Platform and Science \& Technology Support Program in the industrial field of Suqian City of China (Grant No. M201614 \& H201615).

Author Contributions: Aibin Ma and Jinghua Jiang conceived and designed the experiments; Qiuyuan Xie, Huan Liu and Zhaojun Cheng performed the experiments; Qiyuan Xie, Dan Song and Yuchun Yuan analyzed the data; Qiuyuan Xie contributed reagents/materials/analysis tools; Qiuyuan Xie wrote the paper.

Conflicts of Interest: The authors declare no conflict of interest.

\section{References}

1. Winzer, N.; Atrens, A.; Song, G.; Ghali, E.; Dietzel, W.; Kainer, K.U.; Hort, N.; Blawert, C. A Critical Review of the Stress Corrosion Cracking (SCC) of Magnesium Alloys. Adv. Eng. Mater. 2005, 7, 659-693. [CrossRef]

2. Uematsu, Y.; Kakiuchi, T.; Nakajima, M. Stress corrosion cracking behavior of the wrought magnesium alloy AZ31 under controlled cathodic potentials. Mater. Sci. Eng. A 2012, 531, 171-177. [CrossRef]

3. Li, X.C.; Zhang, Y.K.; Chen, J.F.; Lu, Y.L. Effect of laser shock processing on stress corrosion cracking behaviour of AZ31 magnesium alloy at slow strain rate. Mater. Sci. Technol. 2013, 29, 626-630. [CrossRef]

4. Bala Srinivasan, P.; Blawert, C.; Dietzel, W. Effect of plasma electrolytic oxidation coating on the stress corrosion cracking behaviour of wrought AZ61 magnesium alloy. Corros. Sci. 2008, 50, 2415-2418. [CrossRef]

5. Winzer, N.; Atrens, A.; Dietzel, W.; Song, G.; Kainer, K.U. Evaluation of the delayed hydride cracking mechanism for transgranular stress corrosion cracking of magnesium alloys. Mater. Sci. Eng. A 2007, 466, 18-31. [CrossRef]

6. Tomashov, N.D.; Modestova, N.V. Intercrystalline Corrosion and Corrosion of Metals under Stress; L. Hill: London, UK, 1963.

7. Padekar, B.S.; Singh Raman, R.K.; Raja, V.S.; Paul, L. Stress corrosion cracking of a recent rare-earth containing magnesium alloy, EV31A, and a common Al-containing alloy, AZ91E. Corros. Sci. 2013, 71, 1-9. [CrossRef]

8. Pu, Z.; Song, G.L.; Yang, S.; Outeiro, J.C.; Dillion, O.W., Jr.; Puleo, D.A.; Jawahir, I.S. Grain refined and basal textured surface produced by burnishing for improved corrosion performance of AZ31B Mg alloy. Corros. Sci. 2012, 57, 192-201. [CrossRef]

9. Argade, G.R.; Yuan, W.; Kandasamy, K.; Mishra, R.S. Stress corrosion cracking susceptibility of ultrafine grained AZ31. J. Mater. Sci. 2012, 47, 6812-6822. [CrossRef]

10. López, H.F.; Cisneros, M.M.; Mancha, H.; García, O.; Pérez, M.J. Grain size effects on the SCC susceptibility of a nitrogen steel in hot $\mathrm{NaCl}$ solutions. Corros. Sci. 2006, 48, 913-924. [CrossRef]

11. Fairman, L.; Bray, H.J. Transgranular see in Mg-Al alloys. Corros. Sci. 1971, 11, 533-541. [CrossRef]

12. Pelensky, M.; Gallaccio, A. Stress Corrosion of Magnesium Alloys-Environmental Factors. J. Oral Res. 1967, 3, 257-261.

13. Kim, W.J.; Kim, J.K.; Park, T.Y.; Hong, S.I.; Kim, D.I.; Kim, Y.S.; Lee, J.D. Enhancement of strength and superplasticity in a $6061 \mathrm{Al}$ alloy processed by equal-channel-angular-pressing. Metall. Mater. Trans. A 2002, 33, 3155-3164. [CrossRef]

14. Horita, Z.; Fujinami, T.; Nemoto, M.; Langdon, T.G. Improvement of mechanical properties for Al alloys using equal-channel angular pressing. J. Mater. Process. Technol. 2001, 117, 288-292. [CrossRef] 
15. Iwahashi, Y.; Horita, Z.; Nemoto, M.; Langdon, T.G. An investigation of microstructural evolution during equal-channel angular pressing. Acta Mater. 1997, 45, 4733-4741. [CrossRef]

16. Iwahashi, Y.; Furukawa, M.; Horita, Z.; Nemoto, M.; Langdon, T.G. Microstructural characteristics of ultrafine-grained aluminum produced using equal-channel angular pressing. Metall. Mater. Trans. A 1998, 29, 2245-2252. [CrossRef]

17. Gunde, P.; Hänzi, A.C.; Sologubenko, A.S.; Uggowitzer, P.J. High-strength magnesium alloys for degradable implant applications. Mater. Sci. Eng. A 2011, 528, 1047-1054. [CrossRef]

18. Wu, S.; Wang, D.; Deng, C.; Wang, Y. Investigation on Pop-in phenomenon and its causes in CTOD test for weld metal. Trans. China Weld. Inst. 2012, 33, 105-108.

19. Chakrapani, D.G.; Pugh, E.N. The transgranular SCC of a Mg-Al alloy: Crystallographic, fractographic and acoustic-emission studies. Metall. Trans. A 1975, 6, 1155-1163. [CrossRef]

20. Chakrapani, D.G.; Pugh, E.N. Hydrogen embrittlement in a Mg-Al alloy. Metall. Trans. A 1976, 7, $173-178$. [CrossRef]

21. Winzer, N.; Atrens, A.; Dietzel, W.; Song, G.; Kainer, K.U. Comparison of the linearly increasing stress test and the constant extension rate test in the evaluation of transgranular stress corrosion cracking of magnesium. Mater. Sci. Eng. A 2008, 472, 97-106. [CrossRef]

22. Tan, J.C.; Tan, M.J. Dynamic continuous recrystallization characteristics in two stage deformation of Mg-3Al-1Zn alloy sheet. Mater. Sci. Eng. A 2003, 339, 124-132. [CrossRef]

23. Song, D.; Ma, A.B.; Jiang, J.H.; Lin, P.H.; Yang, D.H.; Fan, J.F. Corrosion behaviour of bulk ultra-fine grained AZ91D magnesium alloy fabricated by equal-channel angular pressing. Corros. Sci. 2011, 53, 362-373. [CrossRef]

24. Schino, A.D.; Kenny, J.M. Effects of the grain size on the corrosion behavior of refined AISI 304 austenitic stainless steels. J. Mater. Sci. Lett. 2002, 21, 1631-1634. [CrossRef]

25. Aung, N.N.; Zhou, W. Effect of grain size and twins on corrosion behaviour of AZ31B magnesium alloy. Corros. Sci. 2010, 52, 589-594. [CrossRef]

26. Makar, G.L.; Kruger, J. Corrosion Studies of Rapidly Solidified Magnesium Alloys. J. Electrochem. Soc. 1990, 137, 414-421. [CrossRef]

27. Baliga, C.B.; Tsakiropoulos, P. Development of corrosion resistant magnesium alloys Part 2 Structure of corrosion products on rapidly solidified Mg-16Al alloys. Mater. Sci. Technol. 1993, 9, 513-519. [CrossRef]

28. Song, G.; Atrens, A.; Wu, X.; Zhang, B. Corrosion behaviour of AZ21, AZ501 and AZ91 in sodium chloride. Corros. Sci. 1998, 40, 1769-1791. [CrossRef] 